\title{
Continuous flattening of some pyramids
}

\author{
Chie Nara
}

Chie Nara received her Ph.D. in mathematics from the Ochanomizu University in 1981. She has been a professor at the Tokai University since 2003. Her current research interests include discrete geometry and graph theory.

\section{Introduction}

We use the terminology polyhedron for a polyhedral surface which is permitted to touch itself but not self-intersect, and a polyhedron always can be folded by creases like a piece of paper. A flat folding of a polyhedron is a folding by creases into a multilayered planar shape without self-intersection. The results presented here are related to the following two problems proposed by Erik Demaine et al. ([1], Open Problems 18.1 and 18.3 in [2]).

Problem 1. Can every flat folded state of a polyhedron be reached by a continuous folding process?

Problem 2. Which polyhedra can be flattened according to the straight-skeleton gluing?

E. Demaine et al. (see [2], pp. 281-284) showed that every convex polyhedron possesses a flat folded state obtained by the disk packing method. They also showed the existence of flat folded states obtained by the straight-skeleton gluing for special pyramids and special polyhedra, where they defined the straight skeleton in the three-dimensional space as follows: offset all faces in parallel so that the perpendicular offset distance is equal among

Orangensaft wird häufig in quaderförmigen Kartonverpackungen verkauft. Hat man den Saft ausgetrunken, möchte man den Karton platzsparend im Müll entsorgen, indem man ihn flach in die Ebene faltet. Dies gelingt, ohne den Karton zu bschädigen und sogar ohne die manchmal vorhandenen Leimlaschen zu lösen. In ähnlicher Weise sind auch manche Einkaufstüten aus Papier gefaltet. Es stellt sich sofort die Frage, ob jedes Polyeder in die Ebene gefaltet werden kann, und ob dabei die Ausgangsposition stetig und überschneidungsfrei in die Endposition deformiert werden kann. Die vorliegende Arbeit geht, startend bei dreiseitigen Pyramiden, just dieser Frage nach. 
all inset faces and trace the trajectories of the edges of the polyhedron, forming faces of the straight skeleton which bisect pairs of polyhedron faces.

In this paper, we give a precise definition of the straight-skeleton subdivision and gluing of a convex pyramid $P$ with an $n$-gonal base for $3 \leq n \leq 5$. Then, we prove that there is a continuous flat folding processe for $P$ according to the straight-skeleton gluing. Using this result, we prove that every convex polyhedron is flattened continuously by using the straight-skeleton gluing locally. Finally, we give a remark that each Archimedean polyhedron is continuously flattened by pushing a face toward its opposite face orthogonally.

For each Platonic polyhedron, the existence of a continuous folding process was proved by $\mathrm{J}$. Itoh and the author in [3] by using the property of rhombi, and we refer this continuous folding process as a rhombus method. For general convex polyhedra, J. Itoh, C. Vîlcu and the author in [4] proved, by using the property of cut loci and Alexandrov's gluing theorem, that every convex polyhedron possesses infinitely many continuous flat folding processes (which are referred as a cut locus method), and they also gave a sufficient condition under which any flat folded state of a convex polyhedron can be reached by a continuous folding process, which is a partial answer to Problem 1.

Note that any polyhedron does not change its volume under flexing if shapes of the faces are fixed, which was proved by I. Sabitov [5, 6]. So we need infinite line segments for moving creases to change shapes of some faces and to flatten a polyhedron. The cut locus method requires a lot of portions for moving creases which cover almost the whole surface of a polyhedron ([4]). On the other hand, the rhombus method requires very limited portions of a polyhedron for moving creases. We extend the rhombus method to a method which is applicable to more general situations, and it plays key roles for the proofs of our theorems.

Section 2 is devoted to propose a key lemma (Lemma 1) and its application on kites (convex quadrilaterals with two pairs of equal adjacent sides) (Lemma 2). In Section 3, we define the straight skeleton subdivision and gluing for a pyramid with an $n$-gonal base for $3 \leq n \leq 5$ (Definitions 3-6). We prove main theorems (Theorems $1-3$ ) that every pyramid $P$ with an $n$-gonal base $(3 \leq n \leq 5)$ is continuously flattened by the straight-skeleton gluing so that the $n$-gonal base and one side face have no crease during the folding process. In Section 4 , by applying those main theorems to general convex polyhedra $P$, we prove that $P$ is continuously flattened by local use of the straight-skeleton gluing (Theorem 4).

In Section 5, we give a remark that there is another application of Lemma 2 so that each Archimedean polyhedron is continuously flattened by pushing one face to the opposite face orthogonally.

\section{Folding kites}

For two points $x, y$ in the three-dimensional space $\mathbb{R}^{3}$, we denote by $x y$ the line segment joining those two points, and by dist $(x, y)$ or $|x y|$ the Euclidean distance between those two points. Let $F$ be a two-dimensional surface consisting of polygons in $\mathbb{R}^{3}$ and let $x, y$ be two points of $F$. The intrinsic distance from $x$ to $y$ is defined as the length of shortest paths in $F$ joining $x$ and $y$, and it induces an intrinsic metric on $F$. A mapping from $F$ to a surface $G$ is called an intrinsic isometry if the mapping is isometric with respect to their intrinsic metrics. 
Definition 1. Let $P$ be a polyhedron in $\mathbb{R}^{3}$. We say that a family of polyhedra $\left\{P_{t}: 0 \leq\right.$ $t \leq 1\}$ is a continuous folding process from $P=P_{0}$ to $P_{1}$ if it satisfies the following conditions:

(1) For each $0 \leq t \leq 1$, there is an intrinsic isometry from $P_{t}$ onto $P$.

(2) For each $x \in P$ the mapping $[0,1] \ni \tau \longmapsto P_{\tau}(x) \in\left\{P_{t}(x): 0 \leq t \leq 1\right\}$ is continuous, where $P_{t}(x)$ is the image of $x$ in $P_{t}$.

Moreover, if $P_{1}$ is a flat folded polyhedron, we say that $P$ is flattened by a continuous folding process and we call $P_{1}$ a flat folded polyhedron (or state) of $P$.

For a two-dimensional surface $Q$ in $\mathbb{R}^{3}$ if a family of two-dimensional surfaces $\left\{Q_{t}: 0 \leq\right.$ $t \leq 1\}$ satisfies the corresponding conditions to (1) and (2), we call the family a continuous folding process from $Q=Q_{0}$ to $Q_{1}$.

Definition 2. Let $K=a b c d$ be a kite with $|a b|=|b c|$ and $|a d|=|d c|$ (see Figure 1(1) for example). Choose any point $h$ on $b d$. Fold $K$ in half by a valley fold on $b d$ (the distance of corresponding points to $a$ and $c$ is zero), and then fold by mountain folds on the line segments corresponding to $a h(h c)$, to obtain a flat folding of $K$. We call the resulting shape of $K$ a flat folded kite for $h$ and denoted by $K_{h}$ (see Figure 1(2)). Denote by $a^{\prime}, b^{\prime}, c^{\prime}, d^{\prime}$ and $h^{\prime}$ the images of $a, b, c, d$ and $h$ respectively by the intrinsic isometry from $K$ to $K_{h}$. Define

$$
L_{h}=\operatorname{dist}\left(b^{\prime}, d^{\prime}\right)
$$

In the above process, at first, the distance of corresponding points to $a$ and $c$ decreased to zero, and then the one for $b$ and $d$ decreased to $L_{h}$. Now we show that there is a continuous folding process from $K$ to $K_{h}$ such that those two distances decrease simultaneously. We define a following shape of the kite $K$.

Let $l$ be any given number with $L_{h} \leq l \leq|b d|$. Apply mountain folds to $a h, h c$ and $b h$, and a valley fold to $h d$ so that $\operatorname{dist}\left(b^{\prime}, d^{\prime}\right)=l$ where $x^{\prime}$ means the image of a point $x \in K$ by the intrinsic isometry from $K$ to the resulting shape which is denoted by $S_{l}$. Then such $S_{l}$ is unique except congruence, because $a^{\prime}$ and $c^{\prime}$ are fixed by the three distances from $b^{\prime}, h^{\prime}$, and $d^{\prime}$ (see Figures 1(3) and 1(4)). We call $S_{l}$ a simple folded kite with distance $l$. Define

$$
M_{l}=\operatorname{dist}\left(a^{\prime}, c^{\prime}\right)
$$

Lemma 1. Let $K=$ abcd be a kite in $\mathbb{R}^{3}$ with $|a b|=|b c|$ and $|a d|=|d c|$. Let $h$ be any point on $b d$. For any given numbers $l\left(L_{h} \leq l \leq|b d|\right)$ and $m\left(0 \leq m \leq M_{l}\right)$, there is a point $q$ on hc such that

(1) by applying mountain folds to $\{q b, q c, q d, a h\}$, and valley folds to $\{h b, h q, h d\}$ so that the triangles $\triangle h b q$ and $\triangle h d q$ touch to the triangles $\triangle a b h$ and $\triangle a d h$ respectively (see Figures 1(5)and 1(6)), and that

(2) the resulting figure $K^{\prime}$ satisfies

$$
\operatorname{dist}(f(b), f(d))=l, \operatorname{dist}(f(a), f(c))=m
$$

where $f$ is the intrinsic isometry from $K$ to $K^{\prime}$. We call such $K^{\prime}$ a folded kite with distances $(l, m)$. 


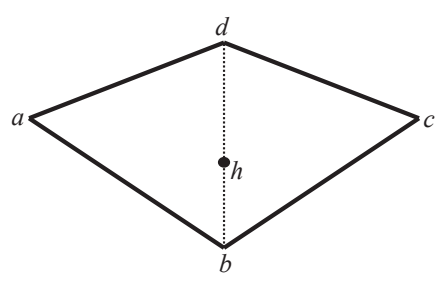

(1)

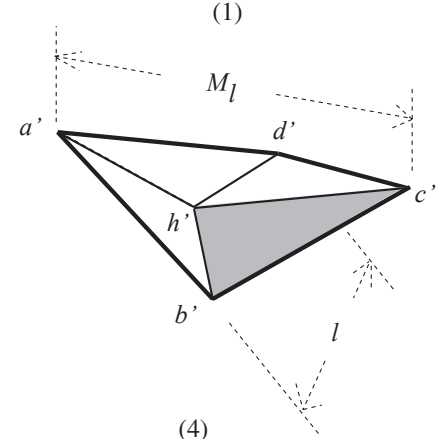

(4)

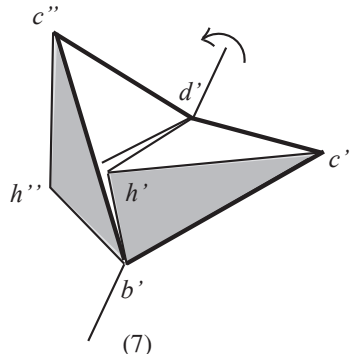

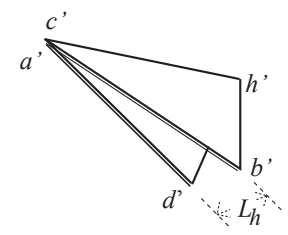

(2)

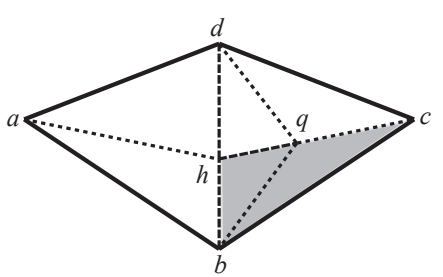

(5)

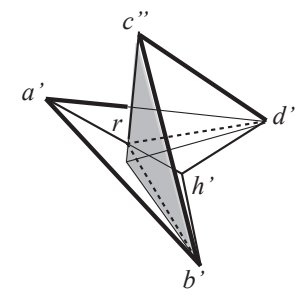

(8)

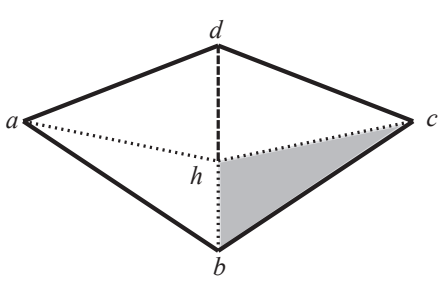

(3)

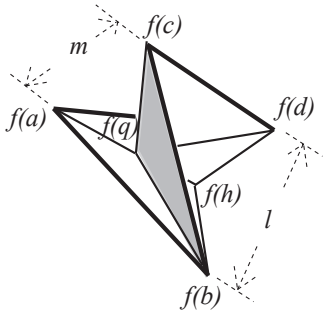

(6)

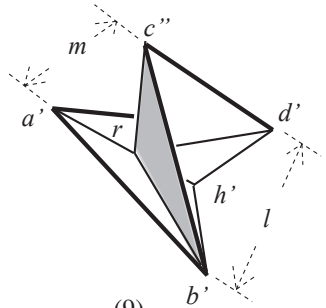

(9)

Fig. 1 How to fold a kite; (1) a kite; (2) a flat folded kite $K_{h}$; (3) creases for mountain folds shown by dotted line segments and a valley fold shown by a bold dotted line segment; (4) a simple folded kite $S_{h}$ with distance $l$; (5) the point $q$ for a given $l, m$ and creases for mountain or valley folds; (6) the shape of the folded kite with distances $(l, m) ;(7)$ a rotated image of the half of $S_{h}$ with $c^{\prime} ;(8)$ the intersection point $r ;(9)$ the folded kite with distances $(l, m)$.

Proof. Let $S_{l}$ be a simple folded kite with the distance $l$ as shown in Figure 1(4) and denote by $x^{\prime} \in S_{l}$ the corresponding point to $x \in K$. Divide $S_{l}$ into halves along $b^{\prime} h^{\prime}$ and $h^{\prime} d^{\prime}$. Those two parts are symmetric to each another about the plane including $\triangle b^{\prime} h^{\prime} d^{\prime}$.

Step 1. Rotate the half part including $c^{\prime}$ about $b^{\prime} d^{\prime}$ so that the distance between $a^{\prime}$ (in the other half part) and $c^{\prime \prime}$ (the rotated point of $c^{\prime}$ ), is the given number $m$ (Figure 1(7)). Denote by $h^{\prime \prime}$ the rotated point of $h^{\prime}$, and by $r$ the intersection of $a^{\prime} h^{\prime}$ and $c^{\prime \prime} h^{\prime \prime}$ (Figure $1(8)$ ). Since the rotated half part and the other half part including $a^{\prime}$ are congruent and symmetric to each other about the plane including $\triangle b^{\prime} r d^{\prime}$, we have $\left|a^{\prime} r\right|=\left|c^{\prime \prime} r\right|$, and hence $\triangle b^{\prime} r h^{\prime} \equiv \triangle b^{\prime} r h^{\prime \prime}$ and $\triangle d^{\prime} r h^{\prime} \equiv \triangle d^{\prime} r h^{\prime \prime}$, where $X \equiv Y$ for subsets $X, Y$ in $\mathbb{R}^{3}$ means that $X$ is congruent to $Y$.

Step 2. Let $q$ be the point on $h c$ in $K$ corresponding to $r$ (Figure 1(5)). Apply mountain folds to $\{q b, q c, q d, a h\}$, and valley folds to $\{h b, h q, h d\}$ so that the triangles $\triangle h b q$ and 
$\triangle h d q$ touch to the triangles $\triangle a b h$ and $\triangle a d h$ respectively. Then we obtain a folded kite with distances $(l, m)$ (Figure 1(9)).

Lemma 2. Let $K=$ abcd be a kite in $\mathbb{R}^{3}$ with $|a b|=|b c|$ and $|a d|=|d c|$. Let $h$ be any point on bd, and let $l_{t} \rightarrow L_{h}\left(L_{h} \leq l_{t} \leq|b d|\right)$ and $m_{t} \rightarrow 0\left(0 \leq m_{t} \leq M_{l_{t}}\right)$ as $t \rightarrow 1$ $(0 \leq t \leq 1)$. There is a continuous folding process $\left\{K_{t}: 0 \leq t \leq 1\right\}$ of folded kites with distances $\left(l_{t}, m_{t}\right)$ so that $K_{0}=K$, and that $K_{1}$ is a flat folded kite for $h$.

Proof. For any fixed $l\left(L_{h} \leq l \leq|b d|\right)$, the point $q$ approaches the point $c$ as $m \rightarrow 0$ where $0 \leq m \leq M_{l}$. So, if $l_{t} \rightarrow L_{h}$ and $m_{t} \rightarrow 0\left(m_{t} \leq M_{l_{t}}\right)$ simultaneously as $t \rightarrow 1$ $(0 \leq t \leq 1)$, there is a continuous folding process of $\left\{K_{t}: 0 \leq t \leq 1\right\}$ of folded kites with $\left(l_{t}, m_{t}\right)$ by Lemma 1 .

Remark. In the continuous process of folded kites with $\left(l_{t}, m_{t}\right)$ for $0 \leq t \leq 1$ shown in the proof of Lemma 2, the point $q$ approaches the point $c$ as $t \rightarrow 1$, and so the creases for mountain folds on $b q$ and $d q$ move from $b h$ and $d h$ to $b c$ and $c d$.

Lemma 1 is an extension of the property of rhombi, shown in [3] where $h$ is fixed as the midpoint of $b d$.

\section{The straight-skeleton subdivision and gluing}

We assume that pyramids are convex through this paper. We define a straight skeleton subdivision of a pyramid $P$ with an $n$-gonal base for $3 \leq n \leq 5$ and show that the pyramid $P$ can be flattened by a continuous folding process according to such a subdivision.

Definition 3. Let $3 \leq n \leq 5$ and let $P$ be a pyramid which has an $n$-gonal base $B=$ $b_{1} b_{2} \cdots b_{n}$, an apex $a$, and side faces $F_{i}(1 \leq i \leq n)$, where $F_{i}$ has the edge $b_{i} b_{i+1}$ and indices are considered modulo $n$ (see Figures 2(1), 3(1), and 4(1)). Let $T$ be its unfolding along $n$ edges adjacent to $a$, and let $a_{i}(1 \leq i \leq n)$ be corresponding points to $a$ in $T$, where $a_{i} \in F_{i}$ (we use the same symbol for the corresponding point or face in $T$ to an original point or face in $P$, except $a$, if there is no fear of confusion) (see Figures 2(2), $3(2)$, and $4(2))$. For each vertex $b_{i}(1 \leq i \leq n)$ there are three faces $\left\{B, F_{i}, F_{i-1}\right\}$ which are adjacent to $b_{i}$, and whose angles at $b_{i}$ are denoted by $\theta_{i}, \xi_{i}$, and $\eta_{i}$ respectively. Define for $i(1 \leq i \leq n)$

$$
\alpha_{i}=\left(\xi_{i}+\eta_{i}-\theta_{i}\right) / 2, \quad \beta_{i}=\xi_{i}-\alpha_{i}, \quad \gamma_{i}=\eta_{i}-\alpha_{i} .
$$

Then for $i(1 \leq i \leq n), \alpha_{i}+\beta_{i}=\xi_{i}, \alpha_{i}+\gamma_{i}=\eta_{i}, \beta_{i}+\gamma_{i}=\theta_{i}$ and

$$
2\left(\alpha_{i}+\beta_{i}+\gamma_{i}\right)=\theta_{i}+\xi_{i}+\eta_{i}=\angle a_{i-1} b_{i} a_{i} .
$$

For each $i(1 \leq i \leq n)$, draw a half-line $l_{i}$ starting at $b_{i}$ toward the interior of $B$ so that $l_{i}$ forms the angles $\beta_{i}$ and $\gamma_{i}$ with the edges $b_{i} b_{i+1}$ and $b_{i} b_{i-1}$ respectively (Figure 2(2)).

Lemma 3. If $P$ is a triangular pyramid, those three half-lines $l_{i}(1 \leq i \leq 3)$ are concurrent.

Proof. Let $p$ be the intersection of $l_{1}$ and $l_{2}$. Let $p_{i}(1 \leq i \leq 3)$ be the mirror image of $p$ about the edge $b_{i} b_{i+1}$ in $T(P)$. Since $\left|a_{1} b_{1}\right|=\left|a_{3} b_{1}\right|,\left|b_{1} p_{1}\right|=\left|b_{1} p\right|=\left|b_{1} p_{3}\right|$, 


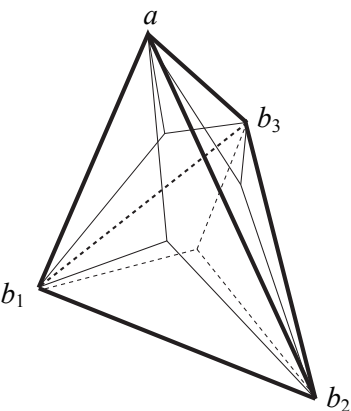

(1)
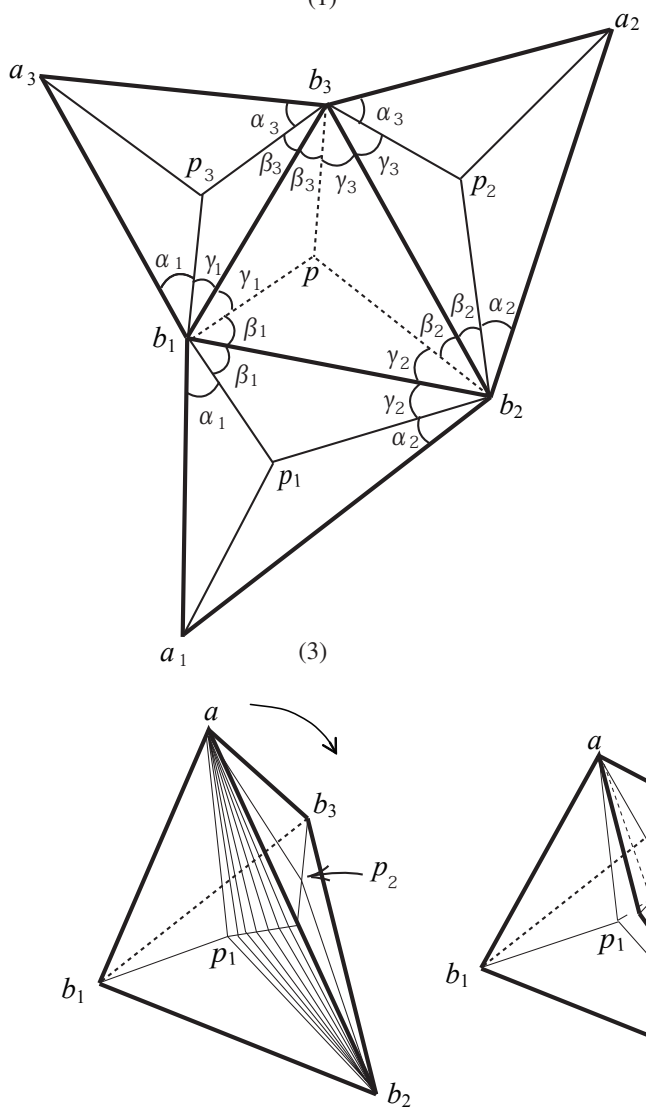

(5)

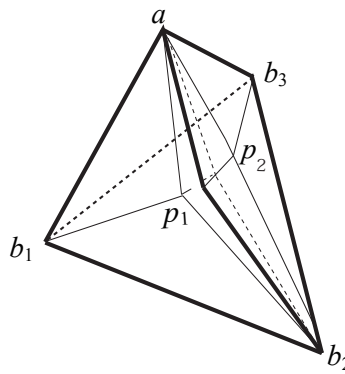

(6)

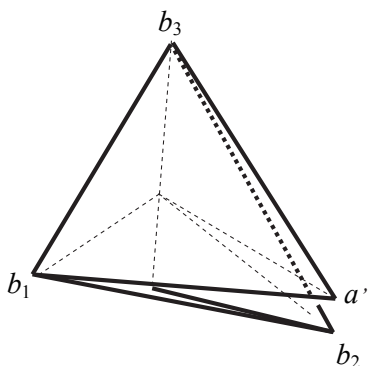

(7)

Fig. 2 (1) A triangular pyramid $P$ and its straight skeleton subdivision; (2) an unfolding of $T$ and half-lines; (3) the corresponding straight skeleton subdivision of its unfolding; (4) the subset $Q$ of $P$ with creases for valley folds on $b_{1} p_{1}$ and $b_{2} p_{2}$; (5) the moving creases for the continuous flat folding process of $P$; (6) $P_{t}$ for some $0<t<1$ in the continuous flat folding process; (7) the flat folded state $P_{1}$. 
and $\angle a_{1} b_{1} p_{1}=\alpha_{1}=\angle a_{3} b_{1} p_{3}$, we have $\triangle a_{1} p_{1} b_{1} \equiv \triangle a_{3} p_{3} b_{1}$ and hence $\left|a_{1} p_{1}\right|=$ $\left|a_{3} p_{3}\right|$. Similarly, we have $\left|a_{1} p_{1}\right|=\left|a_{2} p_{2}\right|$. Therefore $\left|a_{2} p_{2}\right|=\left|a_{3} p_{3}\right|$, which leads $\triangle a_{2} p_{2} b_{3} \equiv \triangle a_{3} p_{3} b_{3}$ by $\left|b_{3} a_{2}\right|=\left|b_{3} a_{3}\right|$ and $\left|b_{3} p_{2}\right|=\left|b_{3} p_{3}\right|$. Since $\angle p_{2} b_{3} p_{3}=2 \theta_{3}$, we have $\angle p_{2} b_{3} a_{2}+\angle p_{3} b_{3} a_{3}=\theta_{3}+\xi_{3}+\eta_{3}-2 \theta_{3}=\xi_{3}+\eta_{3}-\theta_{3}=2 \alpha_{3}$. Hence $\angle a_{2} b_{3} p_{2}=\angle a_{3} b_{3} p_{3}=\alpha_{3}$. Therefore, $\angle b_{1} b_{3} p=\angle b_{1} b_{3} p_{3}=\xi_{3}-\alpha_{3}=\beta_{3}$, and $\angle b_{2} b_{3} p=\eta_{3}-\beta_{3}=\gamma_{3}$, that is, the line $l_{3}$ passes through the point $p$.

Definition 4. Let $P$ be the triangular pyramid discussed in the proof of Lemma 3. Since each face of $P$ includes $p$ or $p_{i}$, draw line segments from such points to the vertices of the face (Figures 2(1) and 2(3)). Then each side edge $a b_{i}(1 \leq i \leq 4)$ in $P$ is a common edge of two congruent triangles which comprise a kite. We call such a subdivision the straight-skeleton subdivision of $P$.

Then all parts in such a subdivision are paired by congruence such that each side edge of $P$ is a diagonal of a kite. If by gluing two parts in each pair (i.e., putting one upon another), we obtain a flat folded state of $P$, we call such state a flat folded state of $P$ with the straight-skeleton gluing.

Theorem 1. Each triangular pyramid $P$ has a continuous folding process $\left\{P_{t}: 0 \leq t \leq 1\right\}$ from $P$ to a flat folded state $P_{1}$ with the straight-skeleton gluing so that the base and one side face of $P$ have no crease during the process.

Proof. Let $Q$ be the subset of $P$ obtained by removing one kite (say $b_{2} p_{1} a p_{2}$ ) from $P$ (see Figure 2(4)). Apply valley folds to line segments $\left\{b_{1} p_{1}, b_{3} p_{2}\right\}$, and rotate the apex $a$ about the edge $b_{1} b_{3}$ toward $b_{2}$ continuously so that it touches the plane including the base $\triangle b_{1} b_{2} b_{3}$. Then $p_{1}$ and $p_{2}$ rotate simultaneously about the edges $b_{1} b_{2}$ and $b_{2} b_{3}$ respectively, and approach to $p \in \triangle b_{1} b_{2} b_{3}$, and hence, $Q$ is flattened continuously.

Let $h$ be the intersection of the edge $a b_{2}$ and the extension of the line segment $b_{3} p_{2}$. Since the quadrilateral $a p_{1} b_{2} p_{2}$ of $P$ is a kite, by Lemma 2 it has a family of folded kites on $p_{2} h$ which comprise a continuous folding process. Hence, $P$ is flattened by a continuous folding process with the straight-skeleton gluing, and $B$ and $F_{3}$ have no crease during the process (see Figures 2(5)-(7)).

Definition 5. Let $P$ be the quadrilateral pyramid discussed in Definition 3 . Let $p$ be the point closer to $b_{1}$ in intersections $l_{1} \cap l_{2}$ and $l_{1} \cap l_{4}$ (either of them if they are equal), say $p=l_{1} \cap l_{2}$. Let $q=l_{3} \cap l_{4}$ (possibly $q=p$ ). Divide the quadrilateral base $B=b_{1} b_{2} b_{3} b_{4}$ into four parts by $\left\{b_{1} p, b_{2} p, b_{3} q, b_{4} q, p q\right\}$ (Figure 3(2)). Divide each side face $F_{i}$ of $P$ by the mirror image of the part including $b_{i} b_{i+1}$ about $b_{i} b_{i+1}$, and by line segments joining $a_{i}$ to the images of $p$ or $q$ (see Figure 4(1) and Figure 4(2)). Then each side edge $a b_{i}(1 \leq i \leq 4)$ in $P$ is a common edge of two congruent triangles which comprise a kite. We call such a subdivision the straight-skeleton subdivision of $P$.

Then all parts in such a subdivision are paired by congruence such that each side edge of $P$ is a diagonal of a kite. If by gluing two parts in each pair, we obtain a flat folded state of $P$, we call such a state a flat folded state of $P$ with the straight-skeleton gluing.

Theorem 2. Any quadrilateral pyramid $P$ has a continuous folding process $\left\{P_{t}: 0 \leq t \leq 1\right\}$ from $P$ to a flat folded state $P_{1}$ with the straight-skeleton gluing so that the quadrilateral base and one side face of $P$ have no crease during the process. 


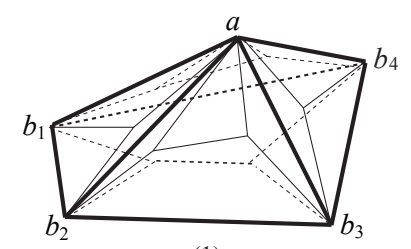

(1)

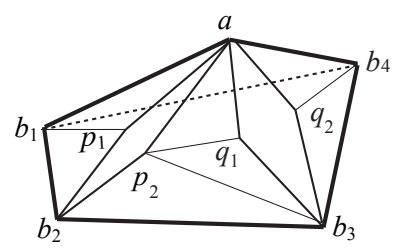

(3)

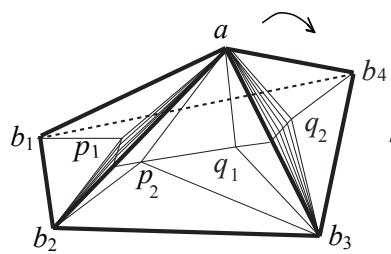

(4)

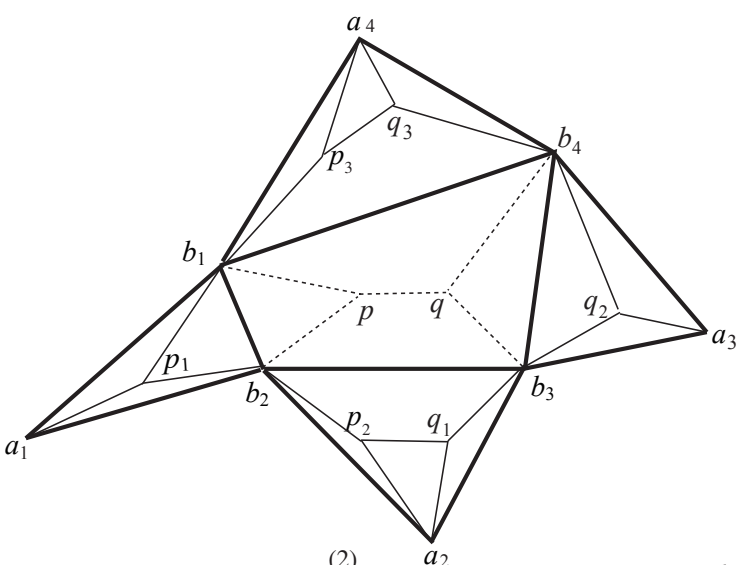

(2)

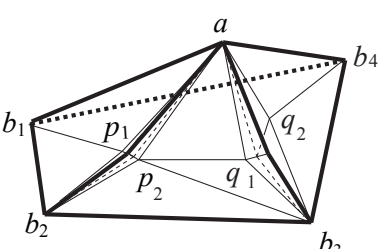

(5)

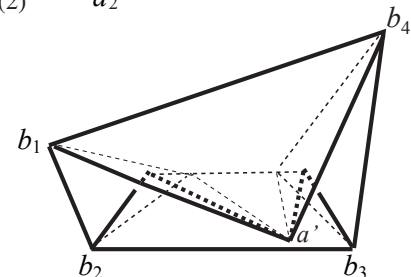

(6)

Fig. 3 (1) A quadrilateral pyramid $P$ and its straight skeleton subdivision; (2) the corresponding straight skeleton subdivision of its unfolding; (3) the subset $Q$ of $P$ with creases for valley folds; (4) the moving creases for the continuous flat folding process of $P$; (5) $P_{t}$ for some $0<t<1$ in the continuous flat folding process; (6) the flat folded state $P_{1}$.

Proof. If $p=q$, the existence of a continuous folding process from $P$ to a flat folded state of $P$ with the straight-skeleton gluing is obtained by a similar argument to the case $n=3$, where we apply Lemma 2 to two kites instead of one. So, we omit the details.

Now assume $p \neq q$. Choose one side face of $P$ which has a quadrilateral in its subdivision. For example, choose $F_{4}=\triangle b_{1} b_{4} a$ in $P\left(\triangle b_{1} b_{4} a_{4}\right.$ in $\left.T\right)$. Let $Q$ be the part of $P$ obtained by removing two kites $a p_{1} b_{2} p_{2}$ and $a q_{1} b_{3} q_{2}$ from $P$, where $Q$ is connected at $a$ (see Figure 5(3)). Apply valley folds to $\left\{b_{1} p_{1}, p_{2} q_{1}, q_{2} b_{4}, p_{2} b_{3}\right\}$ in $Q$. Rotate the apex $a$ about the edge $b_{1} b_{4}$ toward the edge $b_{2} b_{3}$ continuously until it touches the plane including the base $B$. Then, two pairs $\left\{p_{1}, p_{2}\right\}$ and $\left\{q_{1}, q_{2}\right\}$ approach $p$ and $q$ respectively and simultaneously, and hence $Q$ is flattened continuously.

By Lemma 2, each kite which was removed from $P$, is continuously flattened so that the distance between $p_{1}$ and $p_{2}$ as well as the distance between $q_{1}$ and $q_{2}$ decrease to zero. The process is similar to the one for $n=3$, so we omit the details. Hence, $P$ is flattened by a continuous folding process according to the straight-skeleton gluing, and $B$ and $F_{4}$ have no crease during the process (see Figures 4(4)-(6)).

Definition 6. Let $P$ be the pentagonal pyramid discussed in Definition 3. Let $p$ be the closer point to $b_{1}$ in intersections $l_{1} \cap l_{2}$ and $l_{1} \cap l_{5}$ (either of them if they are equal), say $p=l_{1} \cap l_{2}$. Let $q$ be the closer point to $b_{4}$ in intersections $l_{4} \cap l_{5}$ and $l_{4} \cap l_{3}$ (either of them if they are equal), say $q=l_{4} \cap l_{5}$. 
For a point $r$ on the half-line $l_{3}$ in the base $B$ we denote by $r_{1}, r_{2}$, and $r_{3}$ the mirror images of $r$ about $b_{2} b_{3}, b_{3} b_{4}$, and $b_{1} b_{5}$ respectively in $P$. Then we can choose $r$ such that $\left|r_{1} a_{2}\right|=$ $\left|r_{3} a_{5}\right|$, whose existence is as follows. Let $a^{\prime}$ be the mirror image of $a_{5}$ about $b_{1} b_{5}$ in $T$. Fold the face $F_{2}$ with a valley fold along some line segment in $F_{2}$ so that $b_{2} b_{3}$ are fixed and that $a_{2}$ goes to the position of $a^{\prime}$. Then the position of $r$ is obtained as the intersection of $l_{3}$ and the crease for the valley fold. (It may help to consider the quadrilateral pyramid with the base $b_{1} b_{2} b_{3} b_{5}$ and the apex $a$ and apply the continuous folding process to it by Theorem 2, and then the flat folded state indicates the valley fold in $F_{2}$.)

Then $\triangle r_{1} b_{3} a_{2} \equiv \triangle r_{2} b_{3} a_{3}$ by $\left|r_{1} b_{3}\right|=\left|r_{2} b_{3}\right|,\left|a_{2} b_{3}\right|=\left|b_{3} a_{3}\right|$, and $\angle r_{1} b_{3} a_{2}=\angle r_{2} b_{3} a_{3}$. Hence $\left|r_{1} a_{2}\right|=\left|r_{2} a_{3}\right|$ and so

$$
\left|r_{1} a\right|=\left|r_{2} a\right|=\left|r_{3} a\right| \text {. }
$$

Divide $B$ into five parts $B_{i}(1 \leq i \leq 5)$ by $\left\{b_{1} p, b_{2} p, b_{3} r, b_{4} q, b_{5} q, r p, q r\right\}$ so that $B_{i}$ has $b_{i} b_{i+1}$. Divide each side face $F_{i}(1 \leq i \leq 5)$ by the mirror image (denoted by $B_{i}^{\prime}$ ) of $B_{i}$ about $b_{i} b_{i+1}$, and line segments joining vertices of $B_{i}^{\prime}$ to $a\left(a_{i} \in T\right)$ (see Figures $6(1)$ and 6(2)). Then each edge $a b_{i}(1 \leq i \leq 5)$ is a common edge of two congruent triangles which comprise a kite. We call such a subdivision the straight-skeleton subdivision of the pentagonal pyramid $P$.

Then all parts in such a subdivision are paired by congruence such that each side edge of $P$ is a diagonal of a kite. If by gluing two parts in each pair we obtain a flat folded state of $P$, we call such a state a flat folded state of $P$ with the straight-skeleton gluing.

Theorem 3. Any pentagonal pyramid $P$ has a continuous folding process $\left\{P_{t}: 0 \leq t \leq 1\right\}$ from $P$ to a flat folded state $P_{1}$ with the straight-skeleton gluing so that the pentagonal base and one side face of $P$ have no crease during the process.

Proof. If $p=q$, then $p=q=r$, which is similar to the case $n=3, P$ is flattened by a continuous folding process according to the straight-skeleton gluing by applying Lemma 2 to three kites instead of one.

Suppose $p \neq q$. If $p=r$ or $q=r$, which is similar to the case $n=4, P$ is flattened by a continuous folding process according to the straight-skeleton gluing by applying Lemma 2 to three kites instead of two.

Suppose $p \neq r$ and $q \neq r$. Let $e=b_{1} b_{5}$ which is the common edge of two pentagonal parts in the subdivision. Let $K_{i}(1 \leq i \leq 3)$ be kites with the side edge $a b_{i+1}$ as its diagonal, which are disjoint with $e$.

Let $Q$ be the part of $P$ obtained by removing those $K_{i}(1 \leq i \leq 3)$, where $P$ is connected at $a$ (Figure 6(3)). Apply valley folds to $\left\{b_{1} p_{1}, p_{2} r_{1}, r_{2} q_{1}, q_{2} b_{5}, p_{2} b_{3}, q_{1} b_{3}\right\}$ in $Q$. Rotate the apex $a$ about the edge $b_{1} b_{5}$ toward the vertex $b_{3}$ continuously so that it touches the plane including the base $B$. Then, three pairs $\left\{p_{1}, p_{2}\right\},\left\{r_{1}, r_{2}\right\}$, and $\left\{q_{1}, q_{2}\right\}$ approach $p$, $r$, and $q$ respectively and simultaneously, and hence $Q$ is flattened continuously.

By Lemma 2 , kites $K_{i}(1 \leq i \leq 3)$ which are removed from $P$, are continuously flattened so that the distances of pairs $\left\{p_{1}, p_{2}\right\},\left\{r_{1}, r_{2}\right\}$, and $\left\{q_{1}, q_{2}\right\}$ decrease to zero. Hence $P$ is continuously flattened with the straight-skeleton gluing, and $B$ and $F_{5}$ have no crease during the process. 


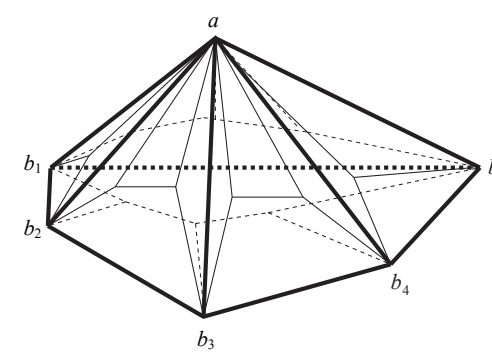

(1)
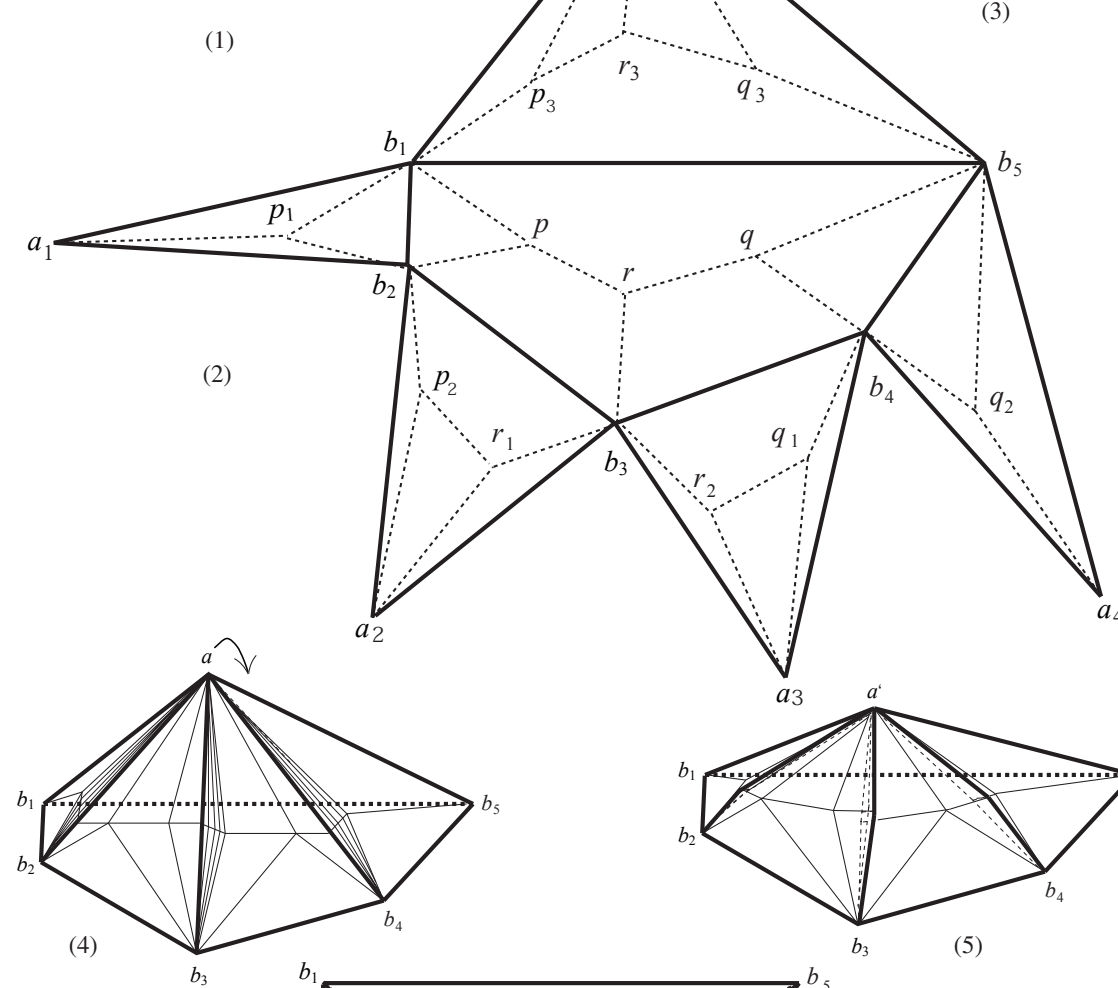

(4)

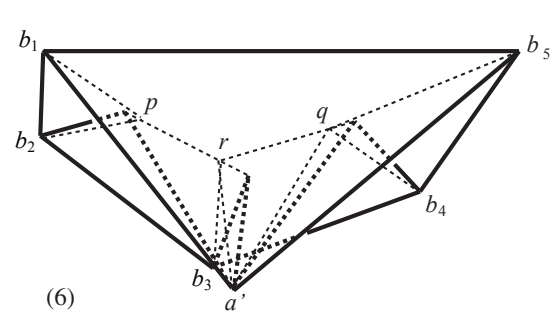

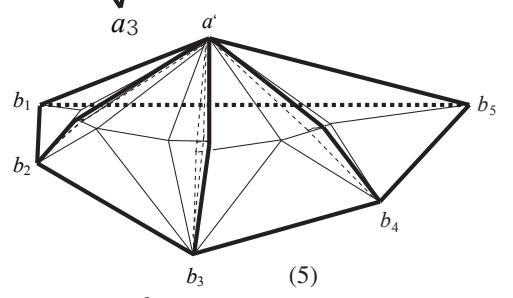

(5)

Fig. 4 (1) A pentagonal pyramid $P$ and its straight skeleton subdivision; (2) the corresponding straight skeleton subdivision of its unfolding; (3) the subset $Q$ of $P$ with creases for valley folds; (4) the moving creases for the continuous flat folding process of $P$; (5) $P_{t}$ for some $0<t<1$ in the continuous flat folding process; (6) the flat folded state $P_{1}$. 


\section{Application to convex polyhedra}

We give an application of the existence of the continuous flat folding process of $n$-gonal pyramids for $3 \leq n \leq 5$ so that the $n$-gonal base has no crease during the process.

Theorem 4. Let $P$ be a convex polyhedron. Then $P$ is flattened continuously by using the straight-skeleton gluing locally.

Proof. We prove Theorem 4 by mathematical induction on the number $n_{P}$ of vertices of a convex polyhedron $P$.

If $n_{p}=4$, then $P$ is a pyramid with a triangular base. Hence it is flattened continuously by the straight-skeleton gluing by Theorem 1 .

Let $k \geq 4$. Assume that any $P$ with $n_{P} \leq k$ vertices can be flattened continuously. Let $P$ be a convex polyhedron with $n_{P}=k+1$ vertices. Since the edge graph of $P$ is planar, there is a vertex $v$ which is incident to at most five edges, which is a well known fact obtained by the Euler formula for a planar graph. Denote the set of incident vertices to $v$ on $P$ by $N_{v}$. Then the number of vertices in $N_{v}$ is at most 5. Let $P^{\prime}$ be the convex hull of the set of vertices of $P$ except $v$.

Consider all planes passing through at least three vertices in $N_{v}$. Choose $H$ among them closest to the vertex $v$. $H$ divides $P$ into a pyramid $T_{v}$ (without a base) and the remaining part of $P$ whose convex hull is denoted by $Q$. Since the boundary of $T_{v}$ is a triangle, a quadrilateral, or a pentagon, we can flatten $T_{v}$ continuously and attach it to $Q$ by Theorems $1-3$.

Let $U$ (possibly an empty set) be the set of all intersections of the plane $H$ and the interior of some edge of $P$ incident to $v$, that is, vertices of $Q$ but not vertices of $P$. Then $U$ has at most two vertices since $N_{v}$ has at most 5 vertices. If $U$ is empty, then $Q$ is congruent to $P^{\prime}$.

If $U$ has only one point $x$, then $x$ is incident to exactly three vertices $w_{i} \in N_{v}(1 \leq i \leq 3)$ in $Q$. Let $T_{x}$ be the pyramid with the apex $x$ and the base $\triangle w_{1} w_{2} w_{3}$. By flattening $T_{x}$ continuously, we obtain a convex polyhedron congruent to $P^{\prime}$.

Suppose $U$ has two points $x$ and $y$. If $x$ and $y$ are not adjacent to each other, then by flattening $T_{x}$ and $T_{y}$ continuously we obtain a convex polyhedra congruent to $P^{\prime}$. If $x$ and $y$ are adjacent to each other, then by flattening $T_{x}$ continuously the degree of $y$ changes to four in the resulting convex polyhedron. So we use for $y$ a similar process used for $v$, and then the resulting polyhedron is congruent to $P^{\prime}$.

Since the number of vertices of $P^{\prime}$ is $k, P^{\prime}$ is flattened continuously by the assumption for $k$ on the mathematical induction. Therefore, $P$ is flattened continuously by using the straight-skeleton gluing locally.

\section{Some remarks}

If a convex polyhedron $Q$ has two parallel faces which includes all vertices of $P$ and which satisfies some additional condition, we can prove by Lemmas 1-2 that $Q$ has a continuous flat folding process so that those two faces are in parallel positions and have no crease during the folding process. By using this fact any Archimedean polyhedron $P$ has a continuous flat folding process so that its specified two parallel faces are parallel and 


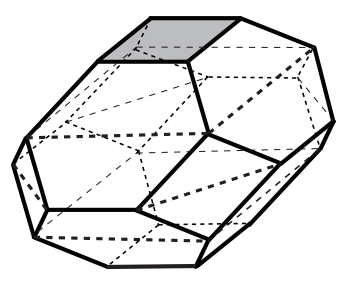

(1)

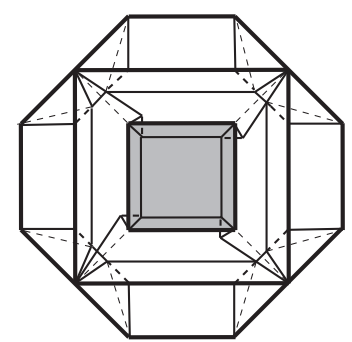

(2)

Fig. 5 (1) Dotted line segments show how to slice the truncated octahedron $P$ by parallel planes; (2) the flat folded state of $P$.

have no crease during the process. The proofs are a little tedious, so we omit them. Figure 5(1) shows how to divide the truncated regular octahedron into parts by parallel planes to have vertices on their boundary and to satisfy conditions. Figure 5(2) shows its flat folded state.

By using the straight-skeleton gluing locally, we may find continuous flat folding processes for non-convex polyhedra. We leave such problem for the future research.

\section{Acknowledgement}

The author would like to express her thanks to Anna Lubiw who showed the author her original model cited in [4], p. 282 and p. 284.

\section{References}

[1] E.D. Demaine, M.D. Demaine, and A. Lubiw, Flattening polyhedra, unpublished manuscript, 2001.

[2] E.D. Demaine and J. O’Rourke, Geometric folding algorithms, Linkages, Origami, Polyhedra, Cambridge University Press (2007).

[3] J. Itoh and C. Nara, Continuous flattening of Platonic polyhedra, Lecture Notes in Computer Science, Proc. CGGA 2010, 7033, Springer-Verlag, Berlin, 108-121 (2011).

[4] J. Itoh, C. Nara, and C. Vîlcu, Continuous flattening of convex polyhedra, Lecture Notes in Computer Science, Proc. EGC 2011, 7579, Springer-Verlag, Berlin, 85-97 (2012).

[5] I. Sabitov, The volume of polyhedron as a function of its metric, Fundam. Prikl. Mat. 2(4), 1235-1246 (1996).

[6] I. Sabitov, The volume as a metric invariant of polyhedra, Discrete Comput. Geom. 20, 405-425 (1998).

\section{Chie Nara}

Liberal Arts Education Center

Aso Campus, Tokai University

Aso, Kumamoto, 869-1404, Japan

e-mail: cnara@ktmail.tokai-u.jp 